\title{
The study of P-glycoprotein A, G-glutamylcysteine synthetase 1 , and aquaglyceroporin 1 genes expression in non-healing zoonotic cutaneous leishmaniasis cases
}

\author{
Seyed Hossein Hejazi ${ }^{\circledR}$, Sedigheh Saberi $^{1}$, Reza Arjmand $^{2}{ }^{\circledR}$, Simindokht Soleimanifard $^{* *}(\mathbb{C}$ \\ ${ }^{1}$ Skin Disease and Leishmaniasis Research Center, Department of Parasitology, School of Medicine Isfahan University of \\ Medical Sciences and Health Services, Isfahan, Iran \\ ${ }^{2}$ Department of Parasitology, School of Medicine, Jundishapur University of Medical Sciences, Ahvaz, Iran \\ ${ }^{3}$ Department of Parasitology and Mycology, School of Medicine, Isfahan University of Medical Sciences and Health Services, \\ Isfahan, Iran
}

*Corresponding Author: Simindokht Soleimanifard, Parasitology and Mycology Department, School of Medicine, Isfahan University of Medical Science, Hezar Jerib Street, Isfahan, Iran. Tel: +989132943369, Email: Soleimanifard@med.mui.ac.ir

\begin{abstract}
Background and aims: Regarding the increasing numbers of clinical antimonial-resistant Leishmaniasis, understanding the reasons for drug resistance is helpful. This study aimed to find the expression level of the genes related to resistance, P-glycoprotein A (PgpA), G-glutamylcysteine synthetase 1 (Gsh1), and aquaglyceroporin 1 (Aqp1) in antimonial-resistant clinical isolates.

Methods: Samples were isolated from leishmaniasis ulcers of 10 non-healing patients and the species were identified by the nestedpolymerase chain reaction (PCR) method. In vitro experiments were performed using the amastigote-macrophage model by $\mathrm{J} 774$ cell line, and in vivo studies were conducted by animal model, the Balb/c mice. Finally, the values of genes expression were determined by quantitative-reverse transcription (q-RT) real-time PCR method and then compared with non-resistant Leishmania major (MRHO/IR/75/ER). Results: Molecular identification showed that all isolated protozoa were $L$. major. The isolated samples from clinical resistant patients represented no increase in expression in the tested resistance genes $(P>0.05)$. Finally, it was found that the lack of improvements in patients was not associated with the increased expression of resistance genes.

Conclusion: In general, no inherent resistance was observed in the tested samples neither a correlation between the healing of lesions and the level of genes expression.

Keywords: Drug resistance, Gene expression, Zoonotic cutaneous leishmaniasis, q-RT PCR
\end{abstract}

Received: 17 February 2021, Accepted: 3 May 2021, ePublished: December 62021

\section{Introduction}

Leishmaniasis is a parasitic disease caused by Leishmania genus of the Trypanosomatidae family in the form of cutaneous (CL), mucocutaneous (MCL), visceral (VL), and post-kala-azar dermal leishmaniasis. The World Health Organization (WHO) identified leishmaniasis as one of the most neglected diseases. Leishmaniasis is endemic in more than 88 countries. Approximately 700 000-1000 000 new cases are annually reported and 26000 to 65000 deaths occur every year. Different species of Leishmania cause cutaneous leishmaniasis, including Leishmania major, Leishmania tropica, and Leishmania aethiopica. In addition, Iran has been identified as an important focus of zoonotic cutaneous leishmaniasis (ZCL) caused by $L$. major $(1,2)$.

Treatment is generally recommended in spite of the selflimiting feature of CL. Treatment can prevent not only scarring but also necrosis and rapid development of lesions following secondary bacterial infections. The pentavalent antimonial compounds Glucantime (meglumine antimoniate) and Pentostam ${ }^{\circledR}$ (sodium stibogluconate) have been the first-line treatment for all forms of leishmaniasis (1). Variations in therapeutic responses to glucantime in previous decades and numerous cases of treatment failure around the world have complicated the role of the first-line drugs in leishmaniasis (3). In the clinical manifestation, resistance to glucantime has been proven by some researchers such Hadighi et al in Iran and Kumar et al in India $(4,5)$. In North Bihar in India, an area with various anthroponotic visceral leishmaniasis, many cases of glucantime resistance have been observed so that up to $60 \%$ of newly diagnosed VL cases in this area do not respond to pentavalent antimonial compounds (6). Previous evidence demonstrated that treatment failure is the result of reduced parasite sensitivity to the antimony compounds (3).

Some studies discovered that the prevalence of parasites becoming resistant to the first-line drug pentavalent antimonial $\left(\mathrm{Sb}^{\mathrm{v}}\right)$ is increasing in several parts of the world and there are wide differences in the sensitivity of various 
$\mathrm{CL}$ isolates to $\mathrm{Sb}^{\mathrm{V}}$ compounds (3). Gene variation and drug resistance in leishmaniasis caused by $L$. tropica have been reported in Iran (7). According to a cross-sectional research study in Mashhad (Iran), 13\% of patients did not respond to glucantime (4).

One of the mechanisms of drug resistance in the parasite is the decrease of drug density. To achieve this goal, the parasite does some mechanisms such as a decrease in receiving the drug, sequestrate, and efflux of the received drug, inhibiting the activation of the drug or degradation of the activated drug by metabolism or analysis (3). Adenosine triphosphate (ATP)-binding cassette proteins $(\mathrm{ABC})$ are present in all organisms and known as the largest superfamily of efflux pumps. Different $\mathrm{ABC}$ classes are explained in Leishmania, one of which is P-glycoprotein A (PgpA), which is a gross membrane protein that acts as an ATP-dependent extraction pump and controls the mechanism of drug efflux and its sequestration (3).

It has been proven that glucantime-resistant Leishmania, as a multidrug resistance protein (MDR) which is a homolog class of $\mathrm{ABC}$, is amplified in an external chromosomal shape. It has been demonstrated that the overexpression of PgpA is an important reason for resistance against glucantime (8).

On the other hand, oxidative stress is one of the $\mathrm{Sb}^{\mathrm{V}}$ mechanisms of action against Leishmania, and the existent of Thiol, as an internal reduction, is important for neutralizing the oxidative action. The laboratory mutants of $\mathrm{Sb}^{\mathrm{V}}$-resistant Leishmania species have shown a clear rise in the intracellular thiols level, including trypanothione (TSH), which is the only major thiol in trypanosomatidae (9). The synthesis of G-glutamyl cysteine synthetase (Gsh), encoding gamma-glutamylcysteine synthetase ( $\gamma$-Gcs), determines the TSH level. Therefore, the level of TSH increases due to the high expression of Gsh. In a study on therapeutic failure isolates, Leishmania (Viannia) guyanensis L. represented the overexpression of Gsh1 (9).

Aquaglyceroporin 1 (Aqp1), as another studied gene, is a member of the superfamily of aquaporin. These are membrane channels that allow the entry of small neutral salts such as glycerol or urea (10). In Leishmania species, Aqp1 is the major route of trivalent antimony entry. It is shown that the rate of Aqp1 expression is in relationship with resistance, thus the overexpression of Aqp1 can affect sensitivity to $\mathrm{Sb}^{\mathrm{V}}$ in Leishmania (11).

Resistance against glucantime in some species of Leishmania such as L. tropica has been proven, and the prevalence of no response of $L$. infantum against glucantime has been reported in recent years (12). In this study, the expression rate of PgpA, Gsh1, and Aqplin phenotypic resistance $\mathrm{ZCl}$, was assayed in vitro and in vivo in comparison to non-resistant L. major (MRHO/ IR/75/ER).

\section{Materials and Methods}

Clinical samples, parasite isolation, and culture

This experimental study was performed by sampling ten non-healing CL patients who failed to recover despite receiving at least one full course of glucantime from health centers in Isfahan and its suburbs. They were positively diagnosed by microscopic observations for leishmaniasis. The parasites were isolated from the lesion by transferring the small amount of them in the Novy-Mac Neal-Nicolle) medium at $24 \pm 1^{\circ} \mathrm{C}$.

After isolation, parasites were transferred to the RPMI 1640 medium, supplemented with $10 \%$ inactivated fetal calf serum (FCS), $100 \mu \mathrm{g} / \mathrm{mL}$ streptomycin, and 100 $\mathrm{IU} / \mathrm{mL}$ penicillin $\mathrm{G}$, and finally cultured at $24 \pm 1^{\circ} \mathrm{C}$. $L$. major (MRHO/IR/75/ER) and L. tropica (MRHO/IR/02/ Mash10), as controls, were also cultured through a similar process.

\section{Species identification}

The species were identified by the conserved region of kinetoplast DNA (kDNA) minicircles and the nested polymerase chain reaction (PCR) method. The total DNA of the isolates and controls was extracted with a commercial kit (GeNet Bio, Seoul, Korea) and according to the manufacturer's instructions. The first and second stages of nested-PCR were performed using CSB7XR: ATT TTT CGC GAT TTT CGC AGA ACG and CSB2XF: CGA GTA GCA GAA ACT CCC GTT CA GC primers in the first step, as well as 13Z: ACT GGG GGT TGG TGT AAA ATA G and LiR: TCG CAG AAC GCC CCT primers in the second one (13).

The PCR products were electrophoresed by applying $1.5 \%$ agarose gel, stained by ethidium bromide, and were observed under a transilluminator. L. major (MRHO/ IR/75/ER) and L. tropica (MHOM/ IR/02/Mash10) were used as standards.

\section{In vitro experiment}

The J774 murine macrophage cell line was purchased from Pasteur Institute of Tehran, Iran and grown at 37 ${ }^{\circ} \mathrm{C}$ and $5 \% \mathrm{CO}_{2}$ in RPMI-1640 supplemented with $20 \%$ heat-inactivated FCS and $100 \mu \mathrm{g} / \mathrm{mL}$ streptomycin and $100 \mathrm{IU} / \mathrm{mL}$ penicillin. After mass production, a scraper was utilized to detach cells from the plates. Subsequent to Neubauer chamber cell counting, $2 \times 10^{6}$ cells/wells were seeded to each $3.5 \mathrm{~cm}^{2}$ well of six-well plates in the mentioned condition for 5-6 days. A well with a $22 \times 22$ coverslip on the bottom was considered for the control group.

The amastigote macrophage model was used to assay gene expression, and two wells were devoted for each sample. One of them was considered for pre-treatment and the other was supposed to treat by the half-maximal inhibitory concentration of glucantime $\left(\mathrm{IC}_{50}\right)$, namely, 7.2 $\mu \mathrm{M}$ (14).

Then, late stationary promastigotes of samples and the standard L. major strain (MRHO/IR/75/ER) were separately added to the grown, adhesive J774 macrophages at a 7:1 ratio of promastigotes/macrophage. The medium was removed 4-6 hours after incubation at $34{ }^{\circ} \mathrm{C}$ and $5 \%$ $\mathrm{CO}_{2}$, and then the cells were washed with phosphate buffer saline, and fresh medium was added as well. The 
plates were incubated at $37^{\circ} \mathrm{C}$ in the presence of $5 \% \mathrm{CO}_{2}$ for an additional 24 hours. Next, the coverslip of the control plate was removed and stained with Giemsa, and the phagocytic act was investigated microscopically.

The total RNA of the phagocytic amastigotes was extracted with RNeasy Mini Kit (QIAGEN, Germany) and then reverse transcribed into cDNA with the Revert Aid First Strand cDNA Synthesis Kit (Fermentas, USA) according to the manufacturer's instructions. The obtained cDNA was used to assess the profiles of RNA expression.

\section{In vivo experiment}

Four six-week-old inbred female $\mathrm{Balb} / \mathrm{c}$ mice were obtained from Tehran Pasteur Institute (Tehran, Iran) and divided into eleven groups each containing 10 animals. For Leishman lesion, 10 groups were inoculated with $2 \times 10^{6}$ stationary promastigotes of 10 different $L$. major samples isolated from the patients, and one group was inoculated with the L. major (MRHO/IR/75/ER) at the base of the tails. Leishmania ulcer was created after 4-5 weeks. Direct sampling and microscopic examination were performed to ensure the existence of parasites in the lesions. For treatment, the mice in each group received glucantime intralesional $(60 \mathrm{mg} / \mathrm{kg} / \mathrm{d})$ for two weeks (14). Sampling was performed before treating the mice, and then RNA was extracted using RNeasy Mini Kit (QIAGEN, Germany) according to the manufacturer's protocol. The isolated RNA was reverse transcribed into cDNA using the Revert Aid First Strand cDNA Synthesis Kit (Fermentas, USA) according to the manufacturer's instructions, and in vivo examinations were continued to evaluate the profiles of RNA expression.

\section{Quantitative-reverse transcriptase real-time PCR}

Quantitative-reverse transcriptase real-time PCR (q-RTPCR) was used to assess the profiles of RNA expression. The total reaction volume was $20 \mu \mathrm{L}$ (contacting SYBR Master 1x: $10 \mu \mathrm{L}$; RNase-free water: $7.5 \mu \mathrm{L}$; Forward and reverse primers each: $0.5 \mu \mathrm{L}$; cDNA: $1.5 \mu \mathrm{L}$ ), and the followed conditions included $95^{\circ} \mathrm{C} / 5$ minutes for 1 cycle, and then $95^{\circ} \mathrm{C} / 10$ seconds and $60^{\circ} \mathrm{C} / 30$ seconds both for 40 cycles. As the template, four genes were analyzed, including PgpA, Gsh1, and Aqp1, and as internal control 18s rRNA. Forward and reverse primers were designed and blasted by the National Center for Biotechnology Information (Table 1).
Gene expression was quantified and analyzed by measuring threshold (CT) and applying the following formula:

$\Delta \Delta \mathrm{CT}=[(\mathrm{CT}$ of treated sample gene $-\mathrm{CT}$ of internal control gene) - (CT of untreated sample gene- CT of internal control gene)]

The results were analyzed by the comparative threshold cycle methods $\left(2^{-\Delta \Delta \mathrm{CT}}\right)$ and appropriate statistical analysis (15). Differences were considered significant at a level of $P \leq 0.05$.

Results

The molecular product of the nested-PCR on agarose gel $1 \%$ showed an amplified band of $560 \mathrm{bp}$, and in comparison with the standards, L. major (MRHO/IR/75/ ER) (560 bp) and L. tropica (MHOM/IR/02/Mash 10, 750 bp), all samples were suggested to be L. major (Figure 1). Figure 2 displays the result of infecting the J774 cell line murine macrophages with promastigotes and the transformed intracellular amastigotes.

The gene expression of PgpA, Gsh1, and Aqp1 was studied for all isolates. Statistical analysis revealed that the expression of the studied genes in the isolates did not increase after exposure to glucantime (Table 2). The expression of these genes in isolates was also the same in comparison with L. major (MRHO/IR/75/ER), and after exposure to glucantime represented no increase in expression both in vitro and in vivo (Table 3 and Figure 3 ). The efficacy of the primers and the PCR was estimated to be $95 \%$.

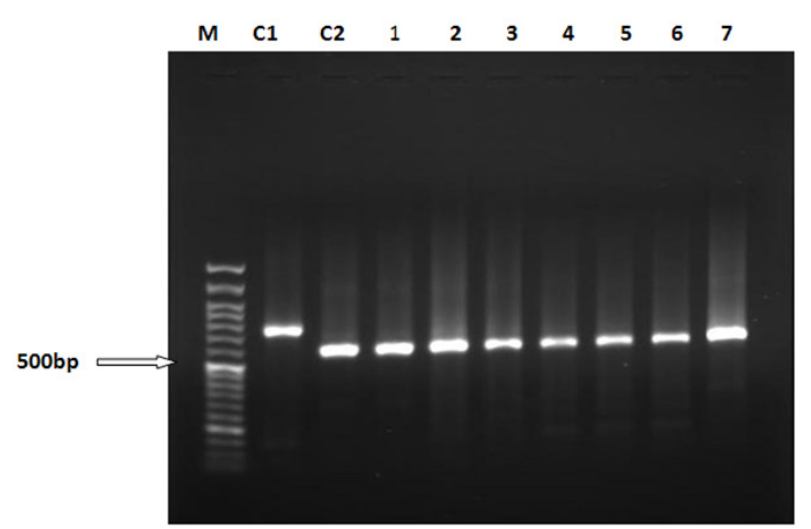

Figure 1. Agarose gel (1\%) electrophoresis of the second phase of nested-PCR product of genomic DNA from patients with resistance phenotype. Note. PCR: Polymerase chain reaction. Lane $\mathrm{M}$ is a 50 bp DNA ladder (Fermentas), C1: DNA from L. tropica (MHOM/IR/02/Mash10), C2: DNA from L. major (MRHO/IR/75/ER). Lanes 1-7 are samples from non-healing individuals.

Table 1. Forward and reverse primers

\begin{tabular}{llll}
\hline Gene & Accession Number & Forward Primer & Reverse Primer \\
\hline \multirow{2}{*}{ Gsh1 } & XM-00168252 & GCTCCACGATGACAC & GGATTCCACACGG \\
& & GGTTACC & CACAGTG \\
PgpA & XM-00168329 & GGTCTCATCTCGCTG & ACGCTCCACGCT \\
& & GCACT & GTCAT \\
Aqp1 & XM-001684934 & TGCCACGATGCCGAG & GTGTTCGTCCTCCTCTTC \\
& & ACTC & CTCAT \\
\hline
\end{tabular}

Note. PgpA: P-glycoprotein A; GSH: G-glutamyl cysteine synthetase; Aqp1: Aquaglyceroporin 1. 
Table 2. The mean expression of PgpA, Gsh1, and Aqp1 genes $(\Delta \mathrm{CT})$ in isolated from patients and standard Leishmania major (MRHO/IR/75/ER) affected by glucantime

\begin{tabular}{|c|c|c|c|c|c|c|}
\hline \multicolumn{4}{|c|}{ Standard L. major (MRHO/IR/75/ER) } & \multicolumn{3}{|c|}{ Patients sp } \\
\hline \multicolumn{7}{|c|}{ In vitro } \\
\hline Gene & Glu- & Glu $^{+}$ & $P$ value & Glu- & $\mathrm{Glu}^{+}$ & $P$ value \\
\hline PgpA & $1.12 \pm 0.01$ & $1.49 \pm 0.01$ & 0.157 & $1.34 \pm 0.29$ & $1.69 \pm 0.76$ & 0.125 \\
\hline GSH & $1.4 \pm 0.01$ & $1.42 \pm 0.01$ & 0.655 & $1.35 \pm 0.2$ & $1.48 \pm 0.25$ & 0.065 \\
\hline AQP1 & $0.53 \pm 0.01$ & $0.5 \pm 0.01$ & 0.182 & $0.57 \pm 0.13$ & $0.83 \pm 0.47$ & 0.097 \\
\hline \multicolumn{7}{|c|}{ In vivo } \\
\hline PgpA & $1.27 \pm 0.007$ & $1.32 \pm 0.007$ & 0.158 & $1.27 \pm 0.2$ & $1.53 \pm 0.74$ & 0.222 \\
\hline Gsh & $1.21 \pm 0.01$ & $1.28 \pm 0.01$ & 0.157 & $1.19 \pm 0.11$ & $1.37 \pm 0.38$ & 0.113 \\
\hline Aqp1 & $0.53 \pm 0.01$ & $0.59 \pm 0.01$ & 0.156 & $0.55 \pm 0.1$ & $0.63 \pm 0.07$ & 0.611 \\
\hline
\end{tabular}

Note. PgpA: P-glycoprotein A; Gsh: G-glutamyl cysteine synthetase; Aqp1: Aquaglyceroporin 1. $P$ value was calculated by paired sample t-test.

\section{Discussion}

Pentavalent antimonial compounds (e.g., meglumine antimoniate or glucantime) are the first-line leishmaniasis treatment (1). However, there are some reports of nonhealing cases with glucantime and the existence of drug resistance (5). Gene expression analysis was used to investigate the non-healing phenotypic cases that have been observed in the Trypanosomatidae family (16). Intracellular amastigotes are the ideal materials and are considered as the gold standard for the leishmaniasis study $(16,17)$.

In this study, L. major was selected because it is endemic in our zone (Isfahan province at the center of Iran), and there are a few gene expression relevant reports about that (2).

Researchers have shown that the expression rate of some kinds of resistance genes, including ornithine decarboxylase, trypanothione reductase (TR), and Aqp1 in resistant and sensitive strains is different, and it was higher in resistant cases $(10,18)$.

Genomic variation in sodium stibogluconate (Pentostam) resistance has been reported in some species

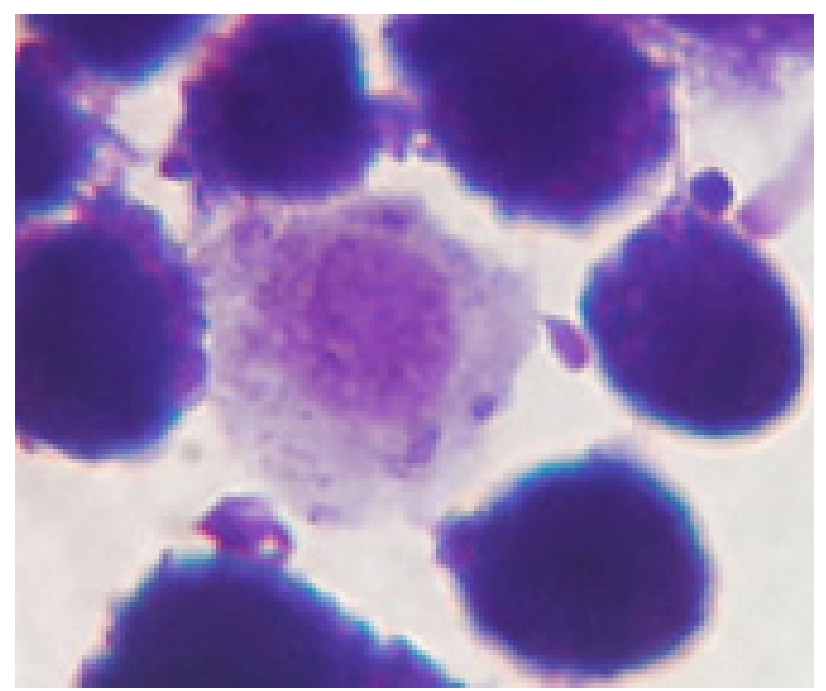

Figure 2. 1774 Cell Line Murine Macrophages Containing Amastigotes (Magnification 1000X). of Leishmania, including L. tropica, which is the cause of anthroponotic CL (19).

The first MDR homologous gene (PgpA) was an extra chromosomal circle (H-circle) amplified in a methotrexate, arsenite-resistant

Leishmania tarentolae promastigote cell line $(19,20)$. A four-fold decrease was observed in the aggregation of antimony in PgpA transfected L. major versus the wild type (20).

In a research study, 97 samples were collected from the ulcers of leishmaniasis patients from some endemic areas of Iran and tested for mutation detection in MDR genes with conformation sensitive gel electrophoresis) and restriction fragment length polymorphism, 10 cases (11\%) among them had a disorder in the sequence of drug-resistance genes (19).

Moreover, in Indian visceral leishmaniasis due to Leishmania donovani, the AQP1 gene demonstrated a higher copy number in antimony-resistant isolates when compared with sensitive isolates. The down-regulation of AQP1 RNA levels was not consistently found in the antimony-resistant isolates. This study indicated that the down-regulation of AQP1 may be one of the mechanisms of antimony resistance and suggested the important role of the AQP1 gene in the generation of antimony-resistant phenotypes although it is not the sole determinant of antimony resistance in the Indian field isolates (21).

The transfection of the PgpA gene and its overexpression led to antimony-resistance to $L$. infantum promastigote and amastigote (22). The amplification of PgpA and Gsh1 genes was observed in the SbIII- and arsenite-resistant mutants (23).

Torres et al clarified that the L. guyanensis isolates of non-healing patients have the overexpression of Gsh1 (24).

In this study, non-healing isolates and susceptible species were investigated, and no significant difference was found in the expression levels of tested genes both in vitro and in vivo. In spite of the achievements of previously mentioned studies, the results of this study indicated that there was no innate resistance against glucantime in $L$. major isolated from non-healing patients. The results 
Table 3. Comparison of relative gene expression data $\left(2^{\Delta \Delta-\mathrm{CT}}\right)$ in standard Leishmania major (MRHO/ER/75/IR) and samples isolated from patients

\begin{tabular}{|c|c|c|c|c|c|c|}
\hline \multicolumn{4}{|c|}{$\begin{array}{c}2^{\Delta \Delta-C} \\
\text { (In vitro) }\end{array}$} & \multicolumn{3}{|c|}{$\begin{array}{c}2^{\Delta \Delta-C}{ }^{T} \\
\text { (In vivo) }\end{array}$} \\
\hline Gene & $\begin{array}{c}\text { L. major } \\
\text { (MRHO/IR/75/ER) }\end{array}$ & $\begin{array}{l}\text { Patients' } \\
\text { isolates }\end{array}$ & $P$ value & $\begin{array}{c}\text { L. major } \\
\text { (MRHO/IR/75/ER) }\end{array}$ & $\begin{array}{l}\text { Patients' } \\
\text { Isolates }\end{array}$ & $P$ value \\
\hline PgpA & $0.77 \pm 0.1$ & $0.78 \pm 0.59$ & 0.371 & $0.83 \pm 0.58$ & $0.96 \pm 0.49$ & 0.573 \\
\hline Gsh & $0.99 \pm 0.007$ & $0.91 \pm 0.18$ & 0.573 & $0.88 \pm 0.1$ & $0.95 \pm .153$ & 0.161 \\
\hline Aqp1 & $1.02 \pm 0.007$ & $0.83 \pm 0.3$ & 0.278 & $0.95 \pm 0.006$ & $0.96 \pm .11$ & 0.361 \\
\hline
\end{tabular}

Note. L. major: Leishmania major; PgpA: P-glycoprotein A; GSH: G-glutamyl cysteine synthetase; AQP1: Aquaglyceroporin 1. P-value was computed by the Mann-Whitney test.

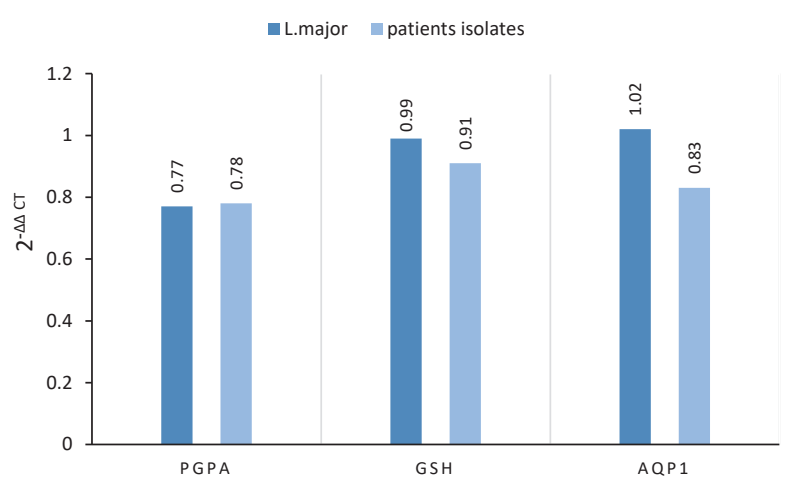

Figure 3. Comparison of $2^{\Delta \Lambda-C T}$ in Leishmania major (MRHO/ER/75/IR) and patients' isolated samples (in vitro exam).

conform to the findings of a study undertaken in Brazil on Leishmania braziliensis and L. guyanensis samples from patients with different responses to antimonial drugs. In the mentioned study, the RNA expression of six genes, by playing a role in antimony metabolism (Aqp1, MRPA, Gsh1, Gsh2, TRYR, and TDR1) was assessed using the q-real-time PCR, and molecular data were compared with the clinical phenotypes. Among the isolates from patients with L. braziliensis with different treatment outcomes, no significant difference was found in the expression levels of the mentioned genes except for Gsh1 (24). Additionally, the findings of some studies in Nepal and Peru showed that there is no significant relationship between the sensitivity of parasites to antimony compounds in vitro and in vivo, indicating that resistance to glucantime is not inherent, and host body factors are highly important in the effectiveness of the drug $(25,26)$.

In a study on the treatment of CL, patients represented significant variations in response to sodium stibogluconate (Pentostam) while no differences to this drug were observed in the sensitivity of $L$. major isolates from patients in the amastigote-macrophage model (27).

Therefore, our data support the suggestion that nonhealing leishmaniasis frequently depends on incomplete irregular intermittent drug use, and this is the major reason for induced resistance.

The results of a research study on 312 patients demonstrated that they failed to recover despite receiving one or more courses of antimony. It was further found that only $26 \%$ of patients were treated according to the WHO guidelines while $72 \%$ of them received the drug fewer than 40 days, $42 \%$ took the drug irregularly, and $36 \%$ stopped the drug receiving arbitrarily (28).
However, treatment failure is a complex interplay between various factors related to either drug, parasite, or host, and thus is not necessarily exclusively linked to drug susceptibility. The quality of host's immunity and genetic, pharmacological factors, drug quality, intrinsic drug properties, treatment duration, and even differences in manufacturers, along with the biological sensitivity of the species of Leishmania, individually and collectively, influence the outcome of treatment. Therefore, due to the mentioned reasons, clinical response to antimony is in the complicated relationship with parasite sensitivity and other factors relevant to the patient or the drug (29)

\section{Conclusion}

Although in many cases, the lack of treatment has been shown to be the innate resistance in different species of Leishmania, no inherent resistance was observed in the examined L. major samples in this study. In addition, no correlation was found between the healing of lesions and the level of gene expression. Accordingly, further investigations are required to find more reasons for the resistance against antimonials. Nonetheless, it is important to mention that probably large-scale drug misuse induces and develops drug resistance, and it is recommended that patients be treated by experienced and skilled physicians according to approved protocols.

\section{Authors' Contribution}

SHH supervised and coordinated the project. SS, SS, and RA performed the experimental section. SS designed and was involved in the preparation of the manuscript.

Conflict of Interests

The authors declare that they have no conflict of interests.

Ethical Approval

The study protocol was approved by the Ethics Committee of Isfahan University of Medical Sciences, Isfahan, Iran (with the code No: IR.IAU.SHK.REC.391042).

\section{Acknowledgment}

This study is the result of the research project (No. 391042, date: 1391) approved by Isfahan University of Medical Sciences. We appreciate the provided financial and spiritual support.

\section{Funding/Support}

This research received no specific grant from funding agencies in the public commercial, or not-for-profit sectors.

\section{References}

1. Pinheiro LCS, de Lourdes G. Ferreira M, Silveira FF, Feitosa 
LM, Boechat N. Synthetic compounds with sulfonamide moiety against leishmaniasis: an overview. Med Chem Res. 2019;28(11):1807-17. doi: 10.1007/s00044-019-02432-3.

2. Norouzinezhad F, Ghaffari F, Norouzinejad A, Kaveh F, Gouya MM. Cutaneous leishmaniasis in Iran: results from an epidemiological study in urban and rural provinces. Asian Pac J Trop Biomed. 2016;6(7):614-9. doi: 10.1016/j. apjtb.2016.05.005

3. Pramanik PK, Alam MN, Roy Chowdhury D, Chakraborti T. Drug resistance in protozoan parasites: an incessant wrestle for survival. J Glob Antimicrob Resist. 2019;18:1-11. doi: 10.1016/j.jgar.2019.01.023.

4. Hadighi R, Boucher P, Khamesipour A, Meamar AR, Roy G, Ouellette $M$, et al. Glucantime-resistant Leishmania tropica isolated from Iranian patients with cutaneous leishmaniasis are sensitive to alternative antileishmania drugs. Parasitol Res. 2007;101(5):1319-22. doi: 10.1007/s00436-007-0638-0.

5. Kumar A, Pandey SC, Samant M. A spotlight on the diagnostic methods of a fatal disease visceral leishmaniasis. Parasite Immunol. 2020;42(10):e12727. doi: 10.1111/pim.12727.

6. Maltezou HC. Drug resistance in visceral leishmaniasis. J Biomed Biotechnol. 2010;2010:617521. doi: 10.1155/2010/617521.

7. Mohammadzadeh M, Behnaz F, Golshan Z. Efficacy of glucantime for treatment of cutaneous leishmaniasis in Central Iran. J Infect Public Health. 2013;6(2):120-4. doi: 10.1016/j. jiph.2012.11.003.

8. Soleimanifard S, Arjmand R, Saberi S, Khamesipour A, Kazemi $M$, Salehi $M$, et al. P-glycoprotein A gene expression in glucantime-resistant and sensitive Leishmania major (MRHO/ IR/75/ER). Iran J Parasitol. 2014;9(3):423-8.

9. Rai S, Bhaskar, Goel SK, Nath Dwivedi U, Sundar S, Goyal $\mathrm{N}$. Role of efflux pumps and intracellular thiols in natural antimony resistant isolates of Leishmania donovani. PLoS One. 2013;8(9):e74862. doi: 10.1371/journal.pone.0074862.

10. Eslami G, Zarchi MV, Moradi A, Hejazi SH, Sohrevardi SM, Vakili $M$, et al. Aquaglyceroporin1 gene expression in antimony resistance and susceptible Leishmania major isolates. J Vector Borne Dis. 2016;53(4):370-4.

11. Plourde M, Ubeda JM, Mandal G, Monte-Neto RL, Mukhopadhyay R, Ouellette M. Generation of an aquaglyceroporin AQP1 null mutant in Leishmania major. Mol Biochem Parasitol. 2015;201(2):108-11. doi: 10.1016/j. molbiopara.2015.07.003.

12. Allahverdiyev AM, Abamor ES, Bagirova M, Baydar SY, Ates SC, Kaya $F$, et al. Investigation of antileishmanial activities of Tio2@Ag nanoparticles on biological properties of $L$. tropica and L. infantum parasites, in vitro. Exp Parasitol. 2013;135(1):55-63. doi: 10.1016/j.exppara.2013.06.001.

13. Noyes HA, Reyburn H, Bailey JW, Smith D. A nested-PCR-based schizodeme method for identifying Leishmania kinetoplast minicircle classes directly from clinical samples and its application to the study of the epidemiology of Leishmania tropica in Pakistan. J Clin Microbiol. 1998;36(10):2877-81. doi: 10.1128/jcm.36.10.2877-2881.1998.

14. Esmaeili J, Mohebali M, Edrissian GH, Rezayat SM, GhaziKhansari M, Charehdar S. Evaluation of miltefosine against Leishmania major (MRHO/IR/75/ER): in vitro and in vivo studies. Acta Med Iran 2008;46(3):191-6.

15. Livak KJ, Schmittgen TD. Analysis of relative gene expression data using real-time quantitative PCR and the 2(-Delta Delta C(T)) Method. Methods. 2001;25(4):402-8. doi: 10.1006/ meth.2001.1262.

16. Gómez Pérez V, García-Hernandez R, Corpas-López V, Tomás AM, Martín-Sanchez J, Castanys S, et al. Decreased antimony uptake and overexpression of genes of thiol metabolism are associated with drug resistance in a canine isolate of Leishmania infantum. Int J Parasitol Drugs Drug Resist. 2016;6(2):133-9. doi: 10.1016/j.ijpddr.2016.04.003.

17. Van den Kerkhof M, Van Bockstal L, Gielis JF, Delputte P, Cos $P$, Maes $L$, et al. Impact of primary mouse macrophage cell types on Leishmania infection and in vitro drug susceptibility. Parasitol Res. 2018;117(11):3601-12. doi: 10.1007/s00436018-6059-4

18. Adaui V, Schnorbusch K, Zimic M, Gutiérrez A, Decuypere $\mathrm{S}$, Vanaerschot $\mathrm{M}$, et al. Comparison of gene expression patterns among Leishmania braziliensis clinical isolates showing a different in vitro susceptibility to pentavalent antimony. Parasitology. 2011;138(2):183-93. doi: 10.1017/ s0031182010001095.

19. Alizadeh R, Hooshyar H, Bandehpor M, Arbabi M, Kazemi F, Talari A, et al. Detection of drug resistance gene in cutaneous leishmaniasis by PCR in some endemic areas of Iran. Iran Red Crescent Med J. 2011;13(12):863-7.

20. Callahan HL, Roberts WL, Rainey PM, Beverley SM. The PGPA gene of Leishmania major mediates antimony (SbIII) resistance by decreasing influx and not by increasing efflux. Mol Biochem Parasitol. 1994;68(1):145-9. doi: 10.1016/01666851(94)00154-5.

21. Maharjan M, Singh S, Chatterjee M, Madhubala R. Role of aquaglyceroporin (AQP1) gene and drug uptake in antimonyresistant clinical isolates of Leishmania donovani. Am J Trop Med Hyg. 2008;79(1):69-75.

22. El Fadili K, Messier N, Leprohon P, Roy G, Guimond C, Trudel $\mathrm{N}$, et al. Role of the ABC transporter MRPA (PGPA) in antimony resistance in Leishmania infantum axenic and intracellular amastigotes. Antimicrob Agents Chemother. 2005;49(5):198893. doi: 10.1128/aac.49.5.1988-1993.2005.

23. Haimeur A, Brochu C, Genest P, Papadopoulou B, Ouellette M. Amplification of the $A B C$ transporter gene PGPA and increased trypanothione levels in potassium antimonyl tartrate (SbIII) resistant Leishmania tarentolae. Mol Biochem Parasitol. 2000;108(1):131-5. doi: 10.1016/s0166-6851(00)00187-0.

24. Torres DC, Adaui V, Ribeiro-Alves M, Romero GA, Arévalo J, Cupolillo E, et al. Targeted gene expression profiling in Leishmania braziliensis and Leishmania guyanensis parasites isolated from Brazilian patients with different antimonial treatment outcomes. Infect Genet Evol. 2010;10(6):727-33. doi: 10.1016/j.meegid.2010.05.006.

25. Rijal S, Yardley V, Chappuis F, Decuypere S, Khanal B, Singh $R$, et al. Antimonial treatment of visceral leishmaniasis: are current in vitro susceptibility assays adequate for prognosis of in vivo therapy outcome? Microbes Infect. 2007;9(4):529-35. doi: 10.1016/j.micinf.2007.01.009.

26. Yardley V, Ortuno N, Llanos-Cuentas A, Chappuis F, Doncker $\mathrm{SD}$, Ramirez L, et al. American tegumentary leishmaniasis: is antimonial treatment outcome related to parasite drug susceptibility? J Infect Dis. 2006;194(8):1168-75. doi: 10.1086/507710.

27. Croft SL, Sundar S, Fairlamb AH. Drug resistance in leishmaniasis. Clin Microbiol Rev. 2006;19(1):111-26. doi: 10.1128/cmr.19.1.111-126.2006.

28. Sundar S, Thakur BB, Tandon AK, Agrawal NR, Mishra CP, Mahapatra TM, et al. Clinicoepidemiological study of drug resistance in Indian kala-azar. BMJ. 1994;308(6924):307. doi: 10.1136/bmj.308.6924.307.

29. Hendrickx S, Guerin PJ, Caljon G, Croft SL, Maes L. Evaluating drug resistance in visceral leishmaniasis: the challenges. Parasitology. 2018;145(4):453-63. doi: 10.1017/ s0031182016002031. 